\title{
DETERMINAÇÃO DE CARBONO ORGÂNICO EM MACROALGAS POR OXIDAÇÃO EM VIA ÚMIDA E TITULAÇÃO POTENCIOMÉTRICA
}

Recebido em: 10/04/2013

Aceito em: $14 / 05 / 2014$

\author{
Leiza Aparecida Souza \\ SERAFIM ${ }^{1}$ \\ Marcelo Friederichs Landim de \\ SOUZA $^{2}$ \\ Cybelle Menolli LONGHINI ${ }^{3}$
}

\section{RESUMO}

A biomassa de produtores primários tais como macroalgas, expressa como concentração de carbono orgânico, é essencial para a realização de balanços de massa e avaliação da dinâmica de ecossistemas marinhos. Este trabalho teve como objetivo a determinação da biomassa de macroalgas através de perda por ignição (PPI), e a comparação da concentração de carbono orgânico estimada através do fator de conversão de PPI e analisada por oxidação em via úmida/titulação potenciométrica, e por combustão catalítica/detector de condutividade térmica.
A análise pela oxidação por via úmida/titulação potenciométrica apresentou alta precisão, e os resultados são comparáveis aos obtidos através da combustão catalítica e detector de condutividade térmica (analisador $\mathrm{CHN}$ ). Estes valores foram correlacionados com a biomassa (PPI), produzindo uma equação diferente do fator PPI x 0,5 encontrado na literatura. As concentrações de carbono orgânico da Chlorophyta Caulerpa racemosa foram superiores aos valores encontrados para as espécies representantes das divisões Phaeophyta e Rhodophyta.

Palavras-chave: perda por ignição, análise química, combustão catalítica, recife de coral

\section{ABSTRACT}

The biomass of primary producers such as macroalgae, express in organic carbon concentration is essential to mass balance studies and an analysis of marine ecosystem dynamics. This study intended to determine the biomass obtained by loss on ignition (LOI), and compare organic carbon in macroalgae estimated by LOI with the concentration obtained by wet oxidation/potentiometric titration and catalytic combustion/thermal conductivity detection methods. The analysis by wet oxidation/potentiometric titration presented high precision, and the results were comparable to those obtained by catalytic combustion/thermal conductivity detection (CNH analyzer). These values were correlated with biomass by LOI, producing an equation different from the LOI $* 0.5$ relation found in the literature. Concentrations of organic carbon of the Chlorophyte Caulerpa racemosa were higher than those of Phaeophyte and Rhodophyte.

Key Words: loss on ignition, chemical analysis, catalytic combustion, coral reefs

\section{INTRODUÇÃO}

As macroalgas são um dos principais produtores primários presentes em ecossistemas recifais. Modificações na biomassa e composição das comunidades de macroalgas podem afetar outros componentes dos ecossistemas marinhos costeiros (MÖRK et al. 2009). A eutrofização

1. Bolsista PIBIC/CNPq. e-mail: leiza_souza@hotmail.com. 2. Laboratório de Biogeoquímica Marinha da UESC. e-mail: marland@bgqmar.net. 3. Programa de Pós-Graduação em Sistemas Aquáticos Tropicais da UESC. e-mail: belle_longhini@yahoo.com.br 
SERAFIM, L. A. S.; SOUZA, M. F. L.; LONGHINI, C. M. Determinação de carbono orgânico em macroalgas por oxidação em via úmida e titulação potenciométrica.

tende a ser o principal evento controlador da biomassa e da assembleia taxonômica de macroalgas, sendo a herbivoria um fator secundário para esta dinâmica (LAPOINTE et al. 2004). Os recifes de Coroa Vermelha (Santa Cruz Cabrália, sul do estado da Bahia) estão sujeitos ao enriquecimento em nutrientes de origem continental (COSTA Jr et al. 2006), tendo como consequência negativa a ocorrência de florescimentos de macroalgas.

O crescimento desordenado das macroalgas prejudica o estabelecimento dos corais escleractíneos devido à competição por espaço na estrutura recifal e pelo sombreamento, diminuindo as taxas fotossintéticas das zooxantelas simbióticas (LAPOINTE et al. 2005). Além destes fatores, interações negativas envolvendo alelopatia também foram observadas na Austrália entre a rodoficea Corallophila huysmansii (Weber-van Bosse) R.E.Norris e o coral Porites cylindrica Dana (JOMPA e MCCOOK 2003).

Estas modificações na estrutura das comunidades implicam em mudanças na armazenagem e fluxos de carbono orgânico, com consequências sobre o metabolismo dos recifes e de áreas adjacentes (BARRÓN et al. 2003). A biomassa de produtores primários, expressa em termos de carbono é fundamental para a elaboração de balanços de massa e análise do funcionamento dos ecossistemas marinhos. Contudo, as técnicas de análise química de carbono orgânico ainda não fazem parte da rotina de grande parte dos laboratórios de Biologia Marinha, Botânica e Ecologia, sendo amplamente utilizada a perda por ignição (PPI) para a obtenção do teor de matéria orgânica e fatores de conversão desta para carbono orgânico. Este trabalho tem como objetivo caracterizar a relação entre a biomassa e a concentração de carbono orgânico em sete espécies de macroalgas comuns na costa brasileira, e comparar as técnicas de análise de carbono orgânico por via úmida e combustão catalítica.

\section{MATERIAL AND METHODS/MATERIAL E METODOS}

Foram coletadas no recife de Coroa Vermelha (Santa Cruz de Cabrália, Bahia) amostras em triplicata de sete espécies de macroalgas pertencentes às divisões Chlorophyta (Caulerpa racemosa (Forsskål) J.Agardh), Phaeophyta (Canistrocarpus cervicornis (Kützing) De Paula \& De Clerck, Feófita 2) e Rhodophyta (Amphiroa fragilíssima (Linnaeus) J.V.Lamouroux, Laurencia filiformis (C.Agardh) Montagne, Sporolithon sp. e Digenia simplex (Wulfen) C. Agardh). As espécies foram selecionadas pela sua representatividade no platô do recife. As amostras foram lavadas e secas em estufa a $60^{\circ} \mathrm{C}$ por 24 horas, e posteriormente maceradas com gral e pistilo. A biomassa livre de cinzas foi determinada pela perda de matéria orgânica por ignição (PPI) após combustão de amostras de 1 grama em um forno mufla a $500^{\circ} \mathrm{C}$, por 10 horas (BROWER e ZAR 1977).

As concentrações de carbono orgânico (CO) foram estimadas a partir da utilização de três métodos: i) O fator de conversão descrito na literatura de 0,5 da PPI (BALE e KENNY 2005); ii) Análise por combustão catalítica e detector de condutividade térmica $(\mathrm{CHN})$ realizada no Centro de Energia Nuclear na Agricultura/USP (CENA) Campus Piracicaba; iii) Oxidação em via úmida com ácido sulfúrico e dicromato de potássio. O segundo método não foi utilizado para as espécies Laurencia filiformis e Sporolithon sp., devido a natureza calcária destas macroalgas o que pode resultar em valores superestimados de carbono orgânico. O terceiro método também não foi empregado para a espécie Sporolithon sp. em decorrência de sua característica morfológica (calcária incrustante).

A oxidação em via úmida com ácido sulfúrico e dicromato de potássio, foi adaptada a partir de dois métodos (ALLEN et al. 1986; CARMOUZE 1994). Alíquotas de 0,01g de cada amostra foram oxidadas em triplicata $(n=63) \mathrm{com} 15 \mathrm{ml}$ de ácido sulfúrico-fosfórico (5:1) e 10 $\mathrm{ml}$ de dicromato de potássio $\left(0,04 \mathrm{~mol} . \mathrm{L}^{-1}\right)$ e colocadas em chapa aquecedora a $100^{\circ} \mathrm{C}$ por uma hora. Posteriormente, realizou-se a titulação potenciométrica dos íons dicromato em excesso com sulfato ferroso amoniacal $0,1 \mathrm{~mol}$. $\mathrm{L}^{-1}$ e eletrodo de anel de Pt. Após a realização da titulação traçou-se a curva do excesso de dicromato ( $\mathrm{mV} \times \mathrm{mL}$ de $\left.\mathrm{Fe}\left(\mathrm{NH}_{4}\right)_{2}\left(\mathrm{SO}_{4}\right)_{2}\right)$ e calculou-se a $2^{a}$ derivada para que fosse determinado o volume de equivalência. A calibração e cálculo do resultado foram realizados através da oxidação em triplicata de $3 \mathrm{~mL}$ de água destilada (branco) e os dois padrões de glicose com concentrações de $750 \mu \mathrm{g} \mathrm{C}^{-1} \mathrm{~L}^{-1}$ e $1500 \mu \mathrm{g}$ C. $\mathrm{L}^{-1}$. A precisão e exatidão do método de oxidação por via úmida foram calculadas, utilizando- 
SERAFIM, L. A. S.; SOUZA, M. F. L.; LONGHINI, C. M. Determinação de carbono orgânico em macroalgas por oxidação em via úmida e titulação potenciométrica.

se como referência os valores de CO obtidos pela análise por combustão catalítica e detector de condutividade térmica no $\mathrm{CHN}$, realizada no CENA.

O teste não paramétrico de Kruskal-Wallis foi aplicado utilizando o programa PAST (HAMMER et al. 2001, http://palaeo-electronica.org/2001_1/past/issue1_01.htm) para avaliar as diferenças entre os métodos analíticos realizados. Quando foi encontrada diferença significativa, o teste a posteriori de Bonferroni foi utilizado a fim de revelar quais métodos diferiram entre si.

\section{RESULTS/RESULTADOS}

Os valores de CO obtidos através do método da oxidação em via úmida apresentaram precisão de $0,02 \mathrm{~g} / \mathrm{g}$ e uma exatidão de $1,1 \%$ em relação aos resultados obtidos pelo CENA. A concentração de carbono orgânico encontrada para todas as macroalgas pelo método da oxidação por via úmida variou numa faixa de 0,14 a $0,22 \mathrm{~g} / \mathrm{g} \mathrm{com}$ média de $0,18 \pm 0,06 \mathrm{~g} / \mathrm{g}$ (Tabela 1).

Table/Tabela 1 - Comparação entre as médias das determinações de carbono orgânico realizadas por via úmida e por combustão $(\mathrm{CHN})$. Teste t pareado $=0,55, p=0,60$.

\begin{tabular}{l|l|l|l}
\hline \multicolumn{1}{c|}{ Amostra } & \multicolumn{1}{c|}{ Via úmida } & \multicolumn{1}{c|}{ CHN } & \multicolumn{1}{c}{ Diferença } \\
\hline Digenia simplex & 0,15 & 0,18 & 0,03 \\
\hline Digenia simplex & 0,14 & 0,15 & 0,00 \\
\hline Digenia simplex & 0,22 & 0,18 & $-0,04$ \\
\hline Digenia simplex & 0,16 & 0,15 & $-0,01$ \\
\hline Feófita 2 & 0,17 & 0,19 & 0,02 \\
\hline Feófita 2 & 0,20 & 0,18 & $-0,02$ \\
\hline Feófita 2 & 0,26 & 0,18 & $-0,08$ \\
\hline Canistrocarpus cervicornis & 0,19 & 0,24 & 0,05 \\
\hline Caulerpa racemosa & 0,28 & 0,33 & 0,05 \\
\hline Amphiroa fragilissima & 0,18 & 0,25 & 0,07 \\
\hline & & & \\
\hline & & média & 0,01 \\
\hline
\end{tabular}

A Chlorophyta C. racemosa apresentou as maiores concentrações de matéria e carbono orgânico (Figura 1). As réplicas das determinações de carbono orgânico por perda por ignição das amostras de $D$. simplex e $C$. cervicornis exibiram um desvio maior do que nas outras espécies analisadas.

As diferentes divisões de macroalgas apresentaram faixas distintas de valores de CO (Chlorophyta, 0,31 $\pm 0,01$; Phaeophyta, 0,19 $\pm 0,04$; Rodophyta, 0,14 $\pm 0,07 \mathrm{~g} \mathrm{CO} \mathrm{.} \mathrm{g}^{-1}$ ) por análise em via úmida. 
SERAFIM, L. A. S.; SOUZA, M. F. L.; LONGHINI, C. M. Determinação de carbono orgânico em macroalgas por oxidação em via úmida e titulação potenciométrica.

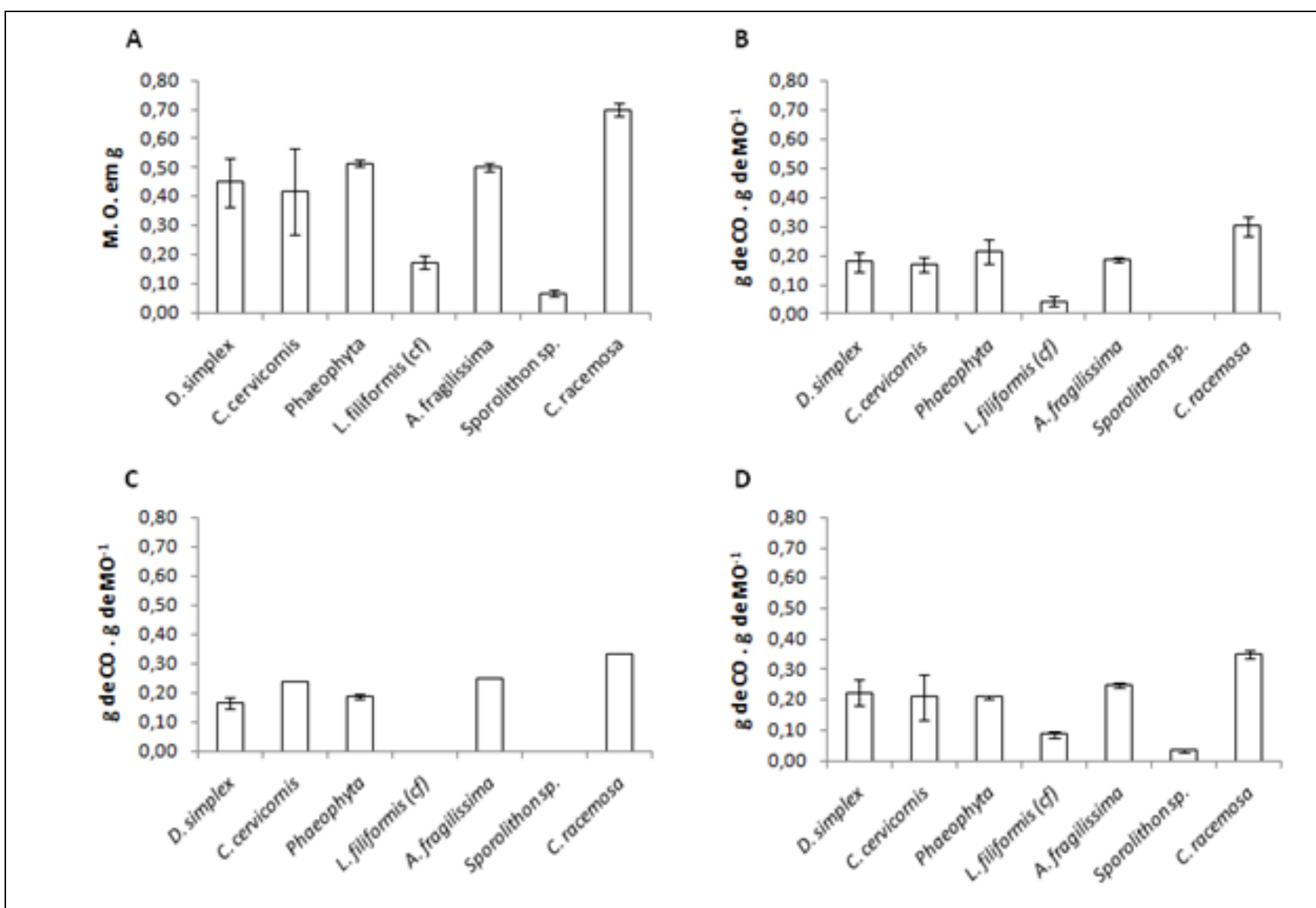

Figura 1 - Teor de matéria orgânica (MO) e concentração de carbono orgânico (CO) para as sete espécies de macroalgas analisadas. A - matéria orgânica obtido por PPI; B CO por oxidação em via úmida; C - CO resultante da análise do CENA; D - CO expresso através do fator de conversão de 0,5 da PPI.

\section{DISCUSSION/DISCUSSÃO}

A análise estatística de Kruskal-Wallis mostrou diferença significativa entre os três métodos utilizados $(p<<<0,01)$. O teste a posteriori de Bonferroni resultou em diferença significativa entre os métodos de conversão de 0,5 da PPI e os métodos por combustão ( $p<<<$ $0,01)$ e oxidação $(p<<<0,01)$. A correlação linear entre os valores de carbono orgânico obtido pela conversão de PPI e a concentração de CO por oxidação em via úmida resultou numa equação com $r^{2}$ igual a 0,87 (Figura 2). Esta equação (CO PPI $=1,71$ CO Oxidação $+0,11$ ) contrasta com a relação de 0,5 descrita na literatura (BALE e KENNY 2005). O uso deste fator de conversão resultou em uma superestimativa da concentração de carbono orgânico em cerca de $4 \%$.

A maior desvantagem das técnicas de análise de carbono orgânico através de oxidação em via úmida com ácido sulfúrico e dicromato de potássio é a produção de resíduos contendo crômio hexavalente, tóxico e carcinogênico em seres humanos. No entanto, estes resíduos podem ser tratados através de técnicas de adsorção (ARULKUMAR et al. 2012) ou de recuperação e reutilização do dicromato (MACHADO et al. 2011). Assim, considerando a adoção de técnicas adequadas de manejo e descarte/reutilização destes resíduos, o método por oxidação em via úmida torna-se uma alternativa viável a ser utilizada nas rotinas de análise em laboratórios que não disponham de equipamentos mais sofisticados e onerosos, tais como o analisador de CHN.

O maior desvio observado nos valores de carbono orgânico por perda por ignição das amostras de $D$. simplex e $C$. cervicornis pode indicar que a temperatura e/ou tempo de calcinação utilizado não é o suficiente para a combustão completa da matéria orgânica nestas espécies. 


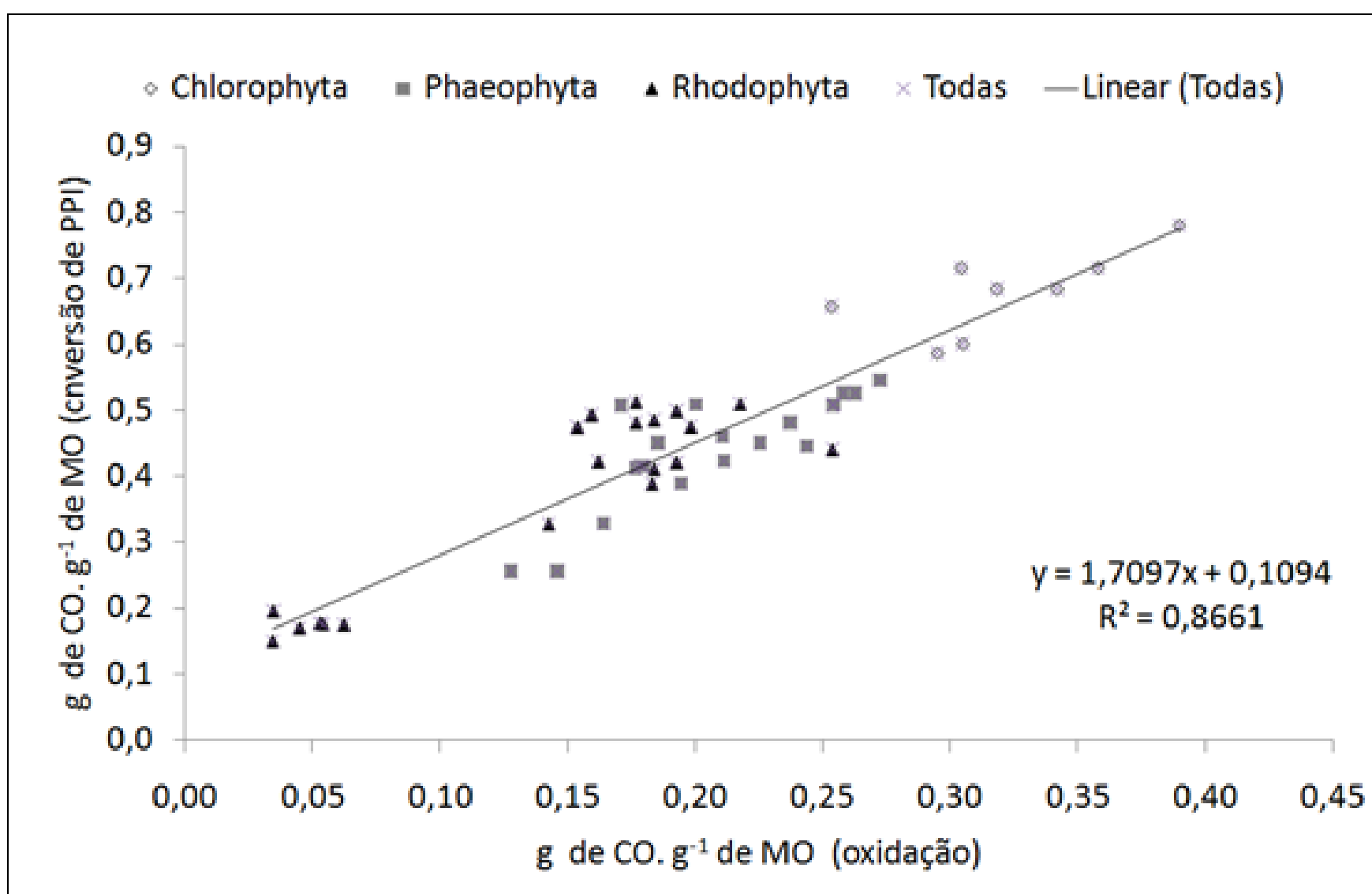

Figura 2 - Correlação entre o teor de carbono orgânico (CO) obtido através da conversão de matéria orgânica por perda por ignição(PPI) e concentração de carbono orgânico por oxidação em via úmida.

Estes resultados apontam para diferenças filogenéticas significativas para as concentrações no percentual de carbono orgânico nas macroalgas, como observado por LAPOINTE et al. (2005). No entanto, os maiores valores de CO foram encontrados para as espécies pertencentes à divisão Phaeophyta $\left(0,26 \pm 0,06 \mathrm{~g} \mathrm{CO} \cdot \mathrm{g}^{-1}\right)$ seguido por Chlorophyta $\left(0,12 \pm 0,05 \mathrm{~g} \mathrm{CO} \cdot \mathrm{g}^{-1}\right)$ e Rhodophyta $\left(0,11 \pm 0,04 \mathrm{~g} \mathrm{CO} \cdot \mathrm{g}^{-1}\right)$.

Este resultado discrepante pode ser devido a pouca representatividade das espécies de macroalgas dos filos Chlorophyta ( 1 espécie) e Phaeophyta ( 2 espécies) utilizadas no presente trabalho. As concentrações de C. racemosa registradas por Lapointe et al. (2005) são cerca de $50 \%$ menores que as registradas em Coroa Vermelha. Este resultado pode estar associado à acumulação de produtos fotossintéticos em função da limitação por fósforo, fator que restringe o aumento da biomassa de macroalgas (LAPOINTE et al. 2005).

\section{CONCLUSION/CONCLUSÃO}

O fator de conversão de perda por ignição para concentração de carbono orgânico descrito na literatura e amplamente utilizado representa uma superestimativa de cerca de $4 \%$ para estas concentrações. A técnica de determinação de carbono orgânico por oxidação em via úmida apresentou resultados semelhantes aos da análise por combustão de detecção por condutividade térmica $(\mathrm{CHN})$, com precisão e exatidão adequados à estudos ecológicos envolvendo balanços de massa.

Como os analisadores de CHN ainda não são disponíveis para grande parte dos pesquisadores que atuam nesta área, a técnica de oxidação por via úmida e titulação potenciométrica representa uma opção de baixo custo e com requerimentos mínimos de infraestrutura laboratorial. 
SERAFIM, L. A. S.; SOUZA, M. F. L.; LONGHINI, C. M. Determinação de carbono orgânico em macroalgas por oxidação em via úmida e titulação potenciométrica.

A espécie de Chlorophyta analisada apresentou concentrações de carbono orgânico superior às de Phaeophyta e Rhodophyta, contrastando com os resultados da literatura.

\section{ACKNOWLEDGEMENTS/AGRADECIMENTOS}

A Dra. Daniela Mariano Lopes da Silva (DCB/UESC) e Luiz Antônio Martinelli (CENA/USP) por viabilizarem a realização das análises de carbono orgânico em analisador CHN; à Dra. Yocie Yoneshigue-Valentin (IB/UFRJ) pela identificação das macroalgas, exceto Feófita 2 para a qual não houve material suficiente para envio e identificação. A primeira autora agradece ao CNPq e à PROPP/UESC pela bolsa PIBIC.

\section{REFERENCES/REFERÊNCIAS BIBLIOGRÁFICAS}

ARULKUMAR, M.; THIRUMALAI, K.; SATHISHKUMAR, P.; PALVANNAN, T. Rapid removal of chromium from aqueous solution using novel prawn shell activated carbon. Chemical

Engineering Journal, V. 185-186, p. 178-186, 2012.

ALLEN, S.E., GRIMSHAW, H.M., ROWLAND, A.P. Chemical Analysis. In: MOORE, P.D. e CHAPMAN, S.B. (Eds.) Methods in Plant Ecology. Oxford: Blackwell Scientific Publications. 1986, Cap. 6, 604 p.

BALE, A.J., KENNY, A.J. Sediment analysis and seabed characterization. In: MCINTYRE, A.D., ELEFTHERIOU, A. (Eds.) Methods for the study of Marine Benthos. Oxford: WileyBlackwell. 2005, Chap. 2, 418 p.

BARRÓN, C.; TMARBA, N.; DUARTE, C.M.; PEDERSEN, M.F.; LINDBLAD, C.; KERSTING, K.; MOY, F.; BOKN, T. High Organic Carbon Export Precludes Eutrophication Responses in Experimental Rocky Shore Communities. Ecosystems, v. 6, p 144-153, 2003.

BROWER J.E., ZAR, J.H. Field and laboratory methods for general Ecology. Dubuque: Brow Company Publishers. 1977, 194 p.

CARMOUZE, J-P. O Metabolismo dos Ecossistemas Aquáticos. Fundamentos teóricos, métodos de estudo e análises químicas. São Paulo: Edgard Blücher/FAPESP, 1994, 253 p.

COSTA JR., O.S.; ATTRILL, M.J.; NIMMO, M. Seasonal and spatial controls on the delivery of excess nutrients to nearshore and offshore coral reefs of Brazil. Journal of Marine Systems, v. 60 , p. $63-74,2006$.

HAMMER, $\varnothing . ;$ HARPER, D.A.T.; RYAN, P.D. PAST: Paleontological statistics software package for education and data analysis. Palaeontologia Electronica, v. 4, n.1, 9pp, 2001.

JOMPA, J.; MCCOOK, L.J. Coral-algal competition: macroalgae with different properties have different effects on corals. Marine Ecology Progress. Series, v. 258, p. 87-95. 2003.

LAPOINTE, B.E; BARILE, P.J.; LITTLER, M.M.; LITTLER, D.S.; BEDFORD, B.J.; GASQUE, C. Macroalgal blooms on southeast Florida coral reefs. I. Nutrient stoichiometry of the invasive green algae Codium isthmocladum in the wider Caribbean indicates nutrient enrichment.

Harmful Algae, v. , p. 1092-1105. 2005.

LAPOINTE, B.E.; BARILE, P.J.; YENTSCHB, C.S.; LITTLER, M.M.; LITTLER, D.S.; KAKUKD, B. The relative importance of nutrient enrichment and herbivory on macroalgal communities near Norman's Pond Cay, Exumas Cays, Bahamas: a "natural" enrichment experiment. Journal of Experimental Marine Biology and Ecology, v. 298, p. 275- 301. 2004.

MACHADO, J.M.C.; OLIVEIRA, L.M.C.P.E.; KAMOGAWA, M.Y. Recycling of chromium in chemical waste from oxidizable carbon determination in organic fertilizer. Química Nova, v. 34, n.1, p. 131-134. 2011.

MÖRK, E.; SJÖÖ, G. L.; KAUTSKY, N.; MCCLANAHAN, T. R. Top-down and bottom-up regulation of macroalgal community structure on a Kenyan reef. Estuarine, Coastal and Shelf Science, v. 84, p. 331-336. 2009. 